\title{
Intraoperative radiotherapy versus whole-breast external beam radiotherapy, and other factors associated with the prognosis of early breast cancer treated with breast-conserving surgery and radiotherapy: a retrospective study from SEER database
}

\author{
Ming-Shuai Sun", Hong-Jin Liu", Yin-Hua Liu, Ling Xu, Jing-Ming Ye \\ Breast Disease Center, Peking University First Hospital, Beijing, China \\ Contributions: (I) Conception and design: MS Sun, HJ Liu; (II) Administrative support: YH Liu, L Xu, JM Ye; (III) Provision of study materials \\ or patients: MS Sun, HJ Liu; (IV) Collection and assembly of data: MS Sun, HJ Liu; (V) Data analysis and interpretation: MS Sun, HJ Liu; (VI) \\ Manuscript writing: All authors; (VII) Final approval of manuscript: All authors. \\ \#These authors contributed equally to this work. \\ Correspondence to: Jing-Ming Ye. Breast Disease Center, Peking University First Hospital, Beijing 100034, China. Email: md_ye@sina.com.
}

\begin{abstract}
Background: This study was aimed to investigate the prognostic factors of early breast cancer treated with breast-conserving surgery (BCS) and radiotherapy. Besides, we focused our attention exclusively on the comparison of the impact on prognosis between intraoperative radiotherapy (IORT) and whole-breast external beam radiotherapy (EBRT).

Methods: An observational cohort study was performed on patients with Tis-2 N0-1 M0 breast cancer from the Surveillance, Epidemiology, and End Results (SEER) database who treated with BCS and radiotherapy. Cox regression analysis, Kaplan-Meier analysis, and propensity score matching (PSM) were used to estimate risk factors for overall survival (OS) and breast cancer-specific survival (BCSS).

Results: Of the 98,614 early breast cancer patients treated with BCS and radiotherapy, 97,164 (98.5\%) patients underwent EBRT and 1,450 (1.5\%) underwent IORT. Multivariable Cox regression analysis showed that early breast cancer patients with age $\geq 65$, poor marital status, lack of medical insurance, histological grade III/IV (SEER 4 grades), high T stage, high N stage, and TNBC were associated with a decreased OS/ BCSS, whereas ER-positive and PR-positive were associated with an improved OS/BCSS. No significant difference was observed in survival between IORT and EBRT groups ( $\mathrm{P}=0.213$ for OS, $\mathrm{P}=0.180$ for BCSS), or between intraoperative beam radiation and intraoperative radioactive implants groups $(\mathrm{P}=0.319$ for OS, $\mathrm{P}=0.972$ for BCSS).

Conclusions: Our study can help clinicians identify patients with poor prognosis after breast-conserving therapy. IORT may be an alternative to EBRT for early breast cancer patients who are unable to complete the long-term postoperative radiation treatment. Beam radiation and radioactive implants are both ideal alternatives for patients who choose IORT.
\end{abstract}

Keywords: Breast cancer; prognosis; breast-conserving surgery (BCS); intraoperative radiotherapy (IORT); SEER database

Submitted Jun 30, 2020. Accepted for publication Sep 14, 2020.

doi: $10.21037 /$ tcr-20-2441

View this article at: http://dx.doi.org/10.21037/tcr-20-2441 


\section{Introduction}

Breast cancer is the most common malignant tumor among women all over the world (1). Since the initial radical mastectomy, breast cancer surgical techniques have been continuously developed. At present, how to preserve the appearance and function of the breast best without affecting the efficacy of standard treatments is receiving increasing attention (2). The NCCN clinical practice guidelines version 2019 recommended breast-conserving surgery (BCS) followed by whole breast radiotherapy as the preferred treatment for early breast cancer (3). Several randomized trials have recommended BCS followed by radiotherapy as the primary local treatment for the majority of stage I-II breast cancer, because it was equivalent to mastectomy on the prognosis (4-7). Although BCS followed by radiotherapy is effective in the treatment of early breast cancer, postoperative local recurrence and death are not rare. In recent years, many prognostic factors such as tumor size, lymph node status, histological grade, hormone receptor status, and biological subtype have been proposed to evaluate the recurrence and survival of early breast cancer treated with BCS and radiotherapy $(8,9)$, while many prognostic factors are still unknown.

The standard method of whole-breast external beam radiotherapy (EBRT), which is administrated by 45-50 Gy in 25-28 fractions to the whole-breast for 5-7 weeks, precludes a considerable proportion of patients from receiving the complete course of radiation treatment (3). To shorten the course of radiotherapy, the fractionation strategies of radiotherapy have been changed from standard schedules (50 Gy in single fractions of 2 Gy) to hypofractionated whole-breast radiation schedules (39-42.9 Gy in single fractions of 2.6-3.3 Gy) (10-12). Recently, the FAST-Forward study further recommended 26 Gy in 5 fractions as a shorter course of radiotherapy, which was expected to become the standard fractionation strategy of postoperative radiotherapy for early breast cancer, because it was non-inferior to $40 \mathrm{~Gy}$ in 15 fractions in both local tumor control and normal tissue effects (13). Additionally, studies reported that $44-86 \%$ of ipsilateral breast tumor recurrences (IBTR) concentrated in the quadrant of primary lesions (14), leading to the emergence of the accelerated partial breast irradiation (APBI) (15). Combining the advantages of hypofractionated radiation and APBI, the concept of intraoperative radiotherapy (IORT) was proposed, which referred to the delivery of single high-dose irradiation to the tumor bed under direct view during the operation. IORT allows a much shorter therapy course, a reduced volume of the irradiated breast, and an improved beauty effect (16-18). At the same time, it also comes at a high cost, which is leading to a higher local recurrence rate (LRR) $(19,20)$. At present, most of the studies are limited to discuss the effect of IORT on the LRR, but the impact on the survival rate of patients has been rarely discussed.

In this retrospective cohort study, we collected a large number of samples from the surveillance, epidemiology, and end results (SEER) database for retrospective analysis to analyze the prognostic factors for early breast cancer patients who underwent BCS and radiotherapy. Different from the previous studies focusing on the recurrence rate (19-21), we took survival rate as the prognostic indicator, went through a long period of follow-up, and fully matched the baseline clinicopathological factors with the help of PSM, to discuss whether using IORT instead of EBRT will affect the survival rate of patients. We present the following article following the STROBE reporting checklist (available at http://dx.doi.org/10.21037/tcr-20-2441).

\section{Methods}

\section{Data acquisition and patient selection}

The SEER database of the National Cancer Institute is a comprehensive population-based, publicly available cancer database, covering about $26 \%$ of the U.S. population. The SEER registry collects all data on patient demographics, cancer incidence, primary tumor sites, cancer histopathology, and survival rates. Our study cohort came from the SEER 18 Regs Custom Data (with additional treatment fields), Nov 2018 Sub (1975-2016 varying), using the SEER*Stat software provided by the National Cancer Institute. Informed consent is unnecessary because SEER data are retrospective and unidentified. The study was conducted in accordance with the Declaration of Helsinki (as revised in 2013).

We collected patients with early breast cancer who underwent BCS and radiotherapy from 2010 to 2016, this date was used as the earliest period of this study because the SEER registry began tracking information about HER-2 status in 2010. The inclusion criteria for this study were as follows: (I) according to the 7th edition of the cancer staging manual of the American Joint Commission on Cancer (AJCC), breast cancer diagnosed with stage Tis-2 N0-1 M0; (II) histopathologically diagnosed as infiltrating duct 
carcinoma (IDC), infiltrating lobular carcinoma (ILC), IDC mixed with other types of carcinoma, or ILC mixed with other types of carcinoma (International Classification of Disease for Oncology code: 8500, 8520, 8521, 8522, 8523, 8524); (III) received BCS, including partial mastectomy/ lumpectomy/re-excision of the biopsy site/segmental mastectomy, etc. (site-specific surgery codes of 20-24); (IV) received intraoperative or postoperative radiotherapy, the IORT mode was beam radiation or radioactive implants, the postoperative radiotherapy mode was beam radiation. The exclusion criteria included: (I) race record unknown, marital status unknown, laterality unknown, insurance record unknown, incomplete surgical information, incomplete radiotherapy information, or incomplete histopathological information; (II) received preoperative radiotherapy, or both intraoperative and postoperative radiotherapy; (III) multifocal or bilateral tumors.

\section{Variables and outcomes}

Demographic and disease characteristics identified in the SEER database and collected in this analysis included: age, year of diagnosis, race, marital status, insurance, laterality, histological grade (SEER 4 grades), histological type, T stage, N stage, ER status, PR status, HER-2 status, subtype, chemotherapy record, radiotherapy record. The primary endpoints of this study were overall survival (OS) and breast cancer-specific survival (BCSS). OS was defined as the duration from the day of diagnosis to death. BCSS was defined as the time between initial diagnosis and death from any cause related to breast cancer.

\section{Statistical analysis}

Categorical data were presented by number (n) and percentage (\%). Pearson chi-square test was used to evaluate the baseline characteristics of patients, Monte Carlo method was adopted when the minimum expected count $<1$, or more than $20 \%$ cells have expected count $<5$. Univariate Cox proportional hazards regression analysis was adopted to find potential prognostic factors, multivariate Cox analysis was used to predict independent risk factors for OS and BCSS, adjusting for variables which were significant in the univariate model. Hazard ratios and $95 \%$ confidence intervals (95\% CIs) were calculated. In the comparison of the survival for patients treated with different radiation strategies, we included demographic and clinicopathological characteristics in the multivariate logistic regression model to perform propensity score matching (PSM) with 1:4 patient pairing and a caliper size of 0.05 , balancing the potential factors that may affect the prognosis of patients. Kaplan-Meier analysis and log-rank test were conducted to investigate the treatment effect on survival. The loss to follow-up or follow-up interruption at the end of the observation was treated as a censoring event. Moreover, we applied a subgroup analysis to test the robustness of our results. All the statistical analyses performed were doublesided, $\mathrm{P} \leq 0.1$ indicated statistical significance in univariate Cox analysis to include more risk factors in multivariate analysis, $\mathrm{P} \leq 0.05$ indicated statistical significance in all other statistical analyses. PSM was performed by R software (version 3.6.3), all other data analyses were performed using SPSS (version 26, IBM Corp.).

\section{Results}

\section{Patient characteristics}

We collected a total of 151,614 patients with early breast cancer who underwent BCS and radiotherapy from 2010 to 2016. A total of 53,000 patients were excluded. Finally, a total of 98,614 patients were included in this study (Figure 1).

Among the 98,614 patients eligible for analysis (Table 1), the median survival time for all patients was 36 months (range, $0-83$ months); 59,650 (60.5\%) patients were less than 65 years old, $80,018(81.1 \%)$ patients were white, $61,791(62.7 \%)$ patients had marital status of married or partner, 88,866 (90.1\%) patients had medical insurance. The proportion of left breast cancer $(50.4 \%)$ was similar to that of right $(49.6 \%) ; 45,397(46.0 \%)$ patients were grade II and 28,168 (28.6\%) were grade I; 81,231 (82.4\%) patients had IDC, which was the most common histological type; 75,742 (76.8\%) patients were T1 stage, while 80,901 (82.0\%) patients were N0 stage. Most patients were ER+ (87.8\%), PR+ (78.6\%), and HER-2- (89.2\%). The most common subtype was HR+/HER-2- (80.3\%). A total of 31,661 (32.1\%) patients received chemotherapy, 97,164 $(98.5 \%)$ patients underwent whole-breast EBRT, and $1,450(1.5 \%)$ patients underwent IORT. The proportion of patients receiving IORT has increased in recent years, from $0.20 \%$ in 2010 to $2.15 \%$ in 2016 .

\section{Radiation strategy and other prognostic factors}

A total of 3,367 deaths occurred, including 1,371 breast cancer-related deaths. Univariate and multivariate Cox regression was used to analyze the prognostic factors for 


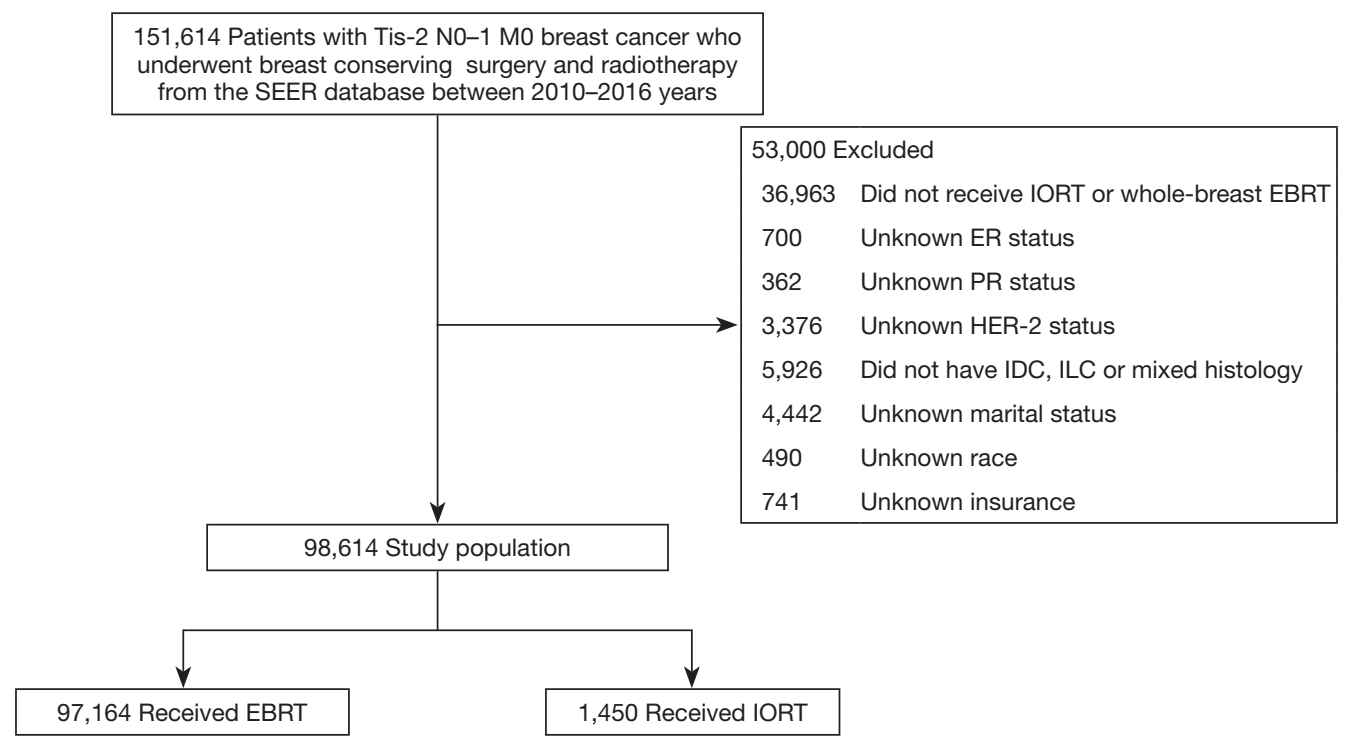

Figure 1 Flow chart of patient selection. SEER, surveillance, epidemiology, and end results; EBRT, external beam radiotherapy; IORT, intraoperative radiotherapy; IDC, infiltrating duct carcinoma; ILC, infiltrating lobular carcinoma.

patients treated with BCS and radiotherapy (Table 2). Univariate Cox regression analysis for OS showed that age, marital status, insurance, histological grade, histological type, T stage, $\mathrm{N}$ stage, ER status, PR status, subtype, and radiation strategy were potential risk factors for OS. The results of the multivariate Cox regression analysis of these factors indicated that patients whose age $\geq 65$ had a significantly decreased OS compared to patients whose age $<65$ (HR, 2.910; 95\% CI, 2.704-3.132; $\mathrm{P}<0.001$ ). Patients who were never married (HR, 1.316; 95\% CI, 1.185-1.462; $\mathrm{P}<0.001$ ) or widowed/divorced/separated (HR, 1.652; 95\% CI, 1.532-1.781; $\mathrm{P}<0.001)$ had a worse OS compared to patients who were married or had a partner. Patients who were uninsured or with any Medicaid had a decreased OS compared to patients who were insured (HR, 1.553; 95\% CI, 1.403-1.719; $\mathrm{P}<0.001)$. Patients with grade III/ IV tumors had a worse OS compared to patients with grade I/II (HR, 1.447; 95\% CI, 1.332-1.573; $\mathrm{P}<0.001)$. Although patients with mixed type tumors had an improved OS compared to IDC (HR, 0.870; 95\% CI, 0.767-0.986; $\mathrm{P}=0.030$ ), we didn't consider the histological type as a risk factor for OS because the overall $\mathrm{P}$ value didn't achieve the remarkable level $(\mathrm{P}=0.066)$. T2 stage (HR, 1.641; 95\% CI, 1.523-1.768; $\mathrm{P}<0.001)$ and $\mathrm{N} 1$ stage $(\mathrm{HR}, 1.455 ; 95 \%$ CI, 1.344-1.576; $\mathrm{P}<0.001)$ were associated with a poorer OS, whereas ER-positive (HR, 0.790; 95\% CI, 0.6650.937; $\mathrm{P}=0.007)$ and $\mathrm{PR}$-positive (HR, 0.829; $95 \% \mathrm{CI}$,
0.746-0.920; $\mathrm{P}<0.001)$ were associated with an increased OS. Patients with TNBC had a worse OS compared to the other three subtypes (HR, 1.262; 95\% CI, 1.066-1.494; $\mathrm{P}=0.007)$. The results of univariate Cox regression analysis for BCSS were similar to those of OS, indicating that age, race, marital status, insurance, laterality, histological grade, histological type, T stage, $\mathrm{N}$ stage, ER status, PR status, subtype, chemotherapy, and radiation strategy were potential risk factors for BCSS. By multivariate Cox regression analysis of these factors, it was found that the risk factors of BCSS were the same as those of OS.

\section{Prognostic value of radiation strategy}

In our study, we further focused our attention exclusively on the comparison of the impact on prognosis between IORT and whole-breast EBRT. After conducting PSM with 1:4 patient pairing to balance the demographic and disease characteristics (Table 3), it was found that almost all characteristics achieved a good match between two groups except marital status and histological type, which was considered acceptable because they were multi-categorical variables. We observed a better BCSS $(\mathrm{P}=0.006)$ and a trend to better OS $(\mathrm{P}=0.070)$ in the IORT group compared to the EBRT group in Kaplan-Meier analysis before PSM, but no significant difference was observed in OS/BCSS between the two groups after PSM $(\mathrm{P}=0.213$ for $\mathrm{OS}, \mathrm{P}=0.180$ for 
Table 1 Basic characteristics of patients with breast cancer who underwent breast-conserving surgery and radiotherapy $(\mathrm{n}=98,614)$

\begin{tabular}{|c|c|}
\hline Characteristics & $\mathrm{N}(\%)$ \\
\hline \multicolumn{2}{|l|}{ Age } \\
\hline$<65$ years & $59,650(60.5)$ \\
\hline$\geq 65$ years & $38,964(39.5)$ \\
\hline \multicolumn{2}{|l|}{ Race } \\
\hline White & $80,018(81.1)$ \\
\hline Black & $9,706(9.8)$ \\
\hline Other & $8,890(9.0)$ \\
\hline \multicolumn{2}{|l|}{ Marital status } \\
\hline Married/unmarried or domestic partner & $61,791(62.7)$ \\
\hline Never married & $13,646(13.8)$ \\
\hline Widowed/divorced/separated & $23,177(23.5)$ \\
\hline \multicolumn{2}{|l|}{ Insurance } \\
\hline Insured/insured no specifics & $88,866(90.1)$ \\
\hline Any Medicaid/uninsured & $9,748(9.9)$ \\
\hline \multicolumn{2}{|l|}{ Laterality } \\
\hline Left & $49,680(50.4)$ \\
\hline Right & $48,934(49.6)$ \\
\hline \multicolumn{2}{|l|}{ Histological grade } \\
\hline I & $28,168(28.6)$ \\
\hline II & $45,397(46.0)$ \\
\hline III & $24,908(25.3)$ \\
\hline IV & $141(0.1)$ \\
\hline \multicolumn{2}{|l|}{ Histological type } \\
\hline IDC & $81,231(82.4)$ \\
\hline ILC & $8,022(8.1)$ \\
\hline Mixed & $9,361(9.5)$ \\
\hline \multicolumn{2}{|l|}{ T stage } \\
\hline Tis & $13(<0.1)$ \\
\hline $\mathrm{T} 1$ & $75,742(76.8)$ \\
\hline $\mathrm{T} 2$ & 22,859 (23.2) \\
\hline \multicolumn{2}{|l|}{$\mathrm{N}$ stage } \\
\hline No & 80,901 (82.0) \\
\hline N1 & $17,713(18.0)$ \\
\hline \multicolumn{2}{|l|}{ ER status } \\
\hline ER- & $12,063(12.2)$ \\
\hline ER+ & $86,551(87.8)$ \\
\hline
\end{tabular}

Table 1 (continued)
Table 1 (continued)

\begin{tabular}{lc}
\hline Characteristics & $\mathrm{N}(\%)$ \\
\hline PR status & $21,073(21.4)$ \\
PR- & $77,541(78.6)$ \\
PR+ & \\
HER-2 status & $87,924(89.2)$ \\
HER-2- & $10,690(10.8)$ \\
HER-2+ & \\
Subtype & $79,233(80.3)$ \\
Luminal A (HR+/HER-2-) & $8,090(8.2)$ \\
Luminal B (HR+/HER-2+) & $2,600(2.6)$ \\
HER-2 enriched (HR-/HER-2+) & $8,691(8.8)$ \\
TNBC (HR-/HER-2-) & \\
Chemotherapy & $66,953(67.9)$ \\
No/unknown & $31,661(32.1)$ \\
Yes & \\
Radiation strategy & $1,450(1.5)$ \\
EBRT & \\
IORT & \\
\hline
\end{tabular}

EBRT, external beam radiotherapy; IORT, intraoperative radiotherapy.

BCSS), in Figure 2 and Table 4. We also didn't observe any prognostic difference in OS between IORT and EBRT groups in further subgroup analysis classified by histological grade, $\mathrm{T}$ stage, $\mathrm{N}$ stage, and molecular subtype (Figure 3). We didn't carry out the subgroup analysis of BCSS because the amount of breast cancer-specific deaths was too small.

\section{Prognostic value of IORT mode}

IORT methods included beam radiation and radioactive implants, so we further compared the survival between these two methods, and we observed no prognostic difference in OS/BCSS between the beam radiation group and the radioactive implants group in Kaplan-Meier analysis $(\mathrm{P}=0.319$ for OS, $\mathrm{P}=0.972$ for BCSS, Figure 4 and Table 5).

\section{Discussion}

BCS for breast cancer is a breakthrough in the history of surgery, and the postoperative adjuvant radiotherapy is an important part of the treatment. NCCN clinical practice 


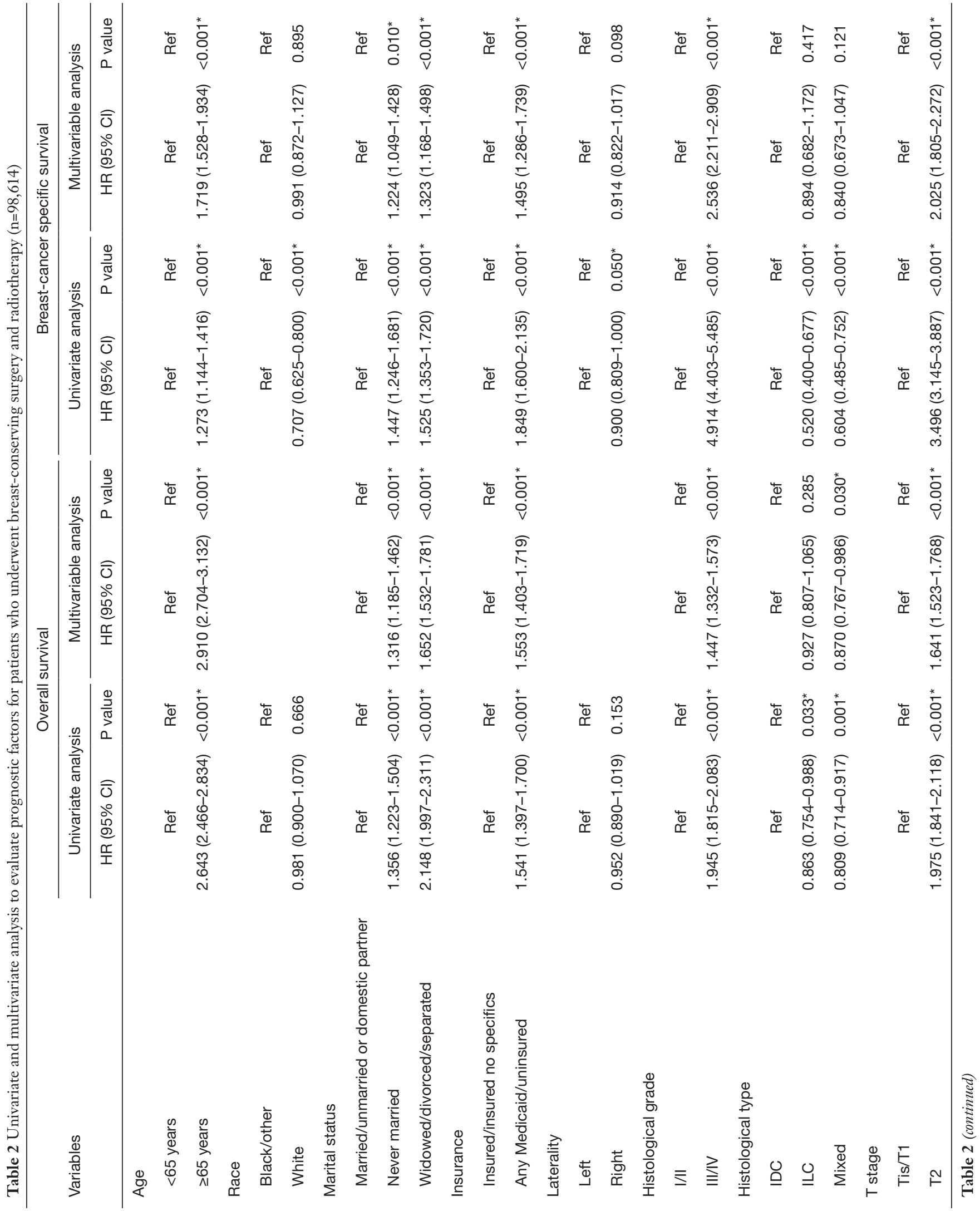




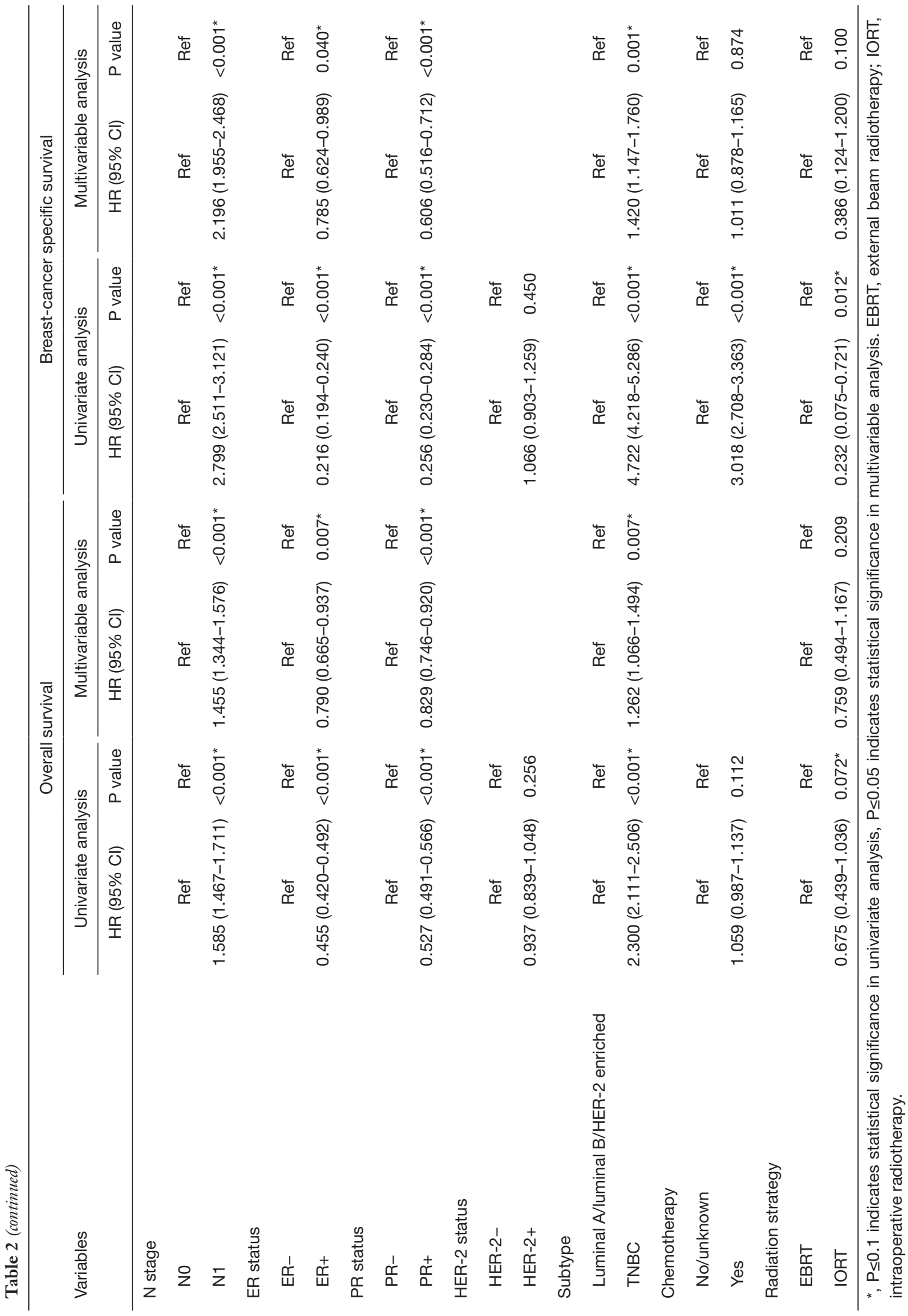


Table 3 Clinicopathological characteristics of patients who underwent breast-conserving surgery and radiotherapy before and after PSM

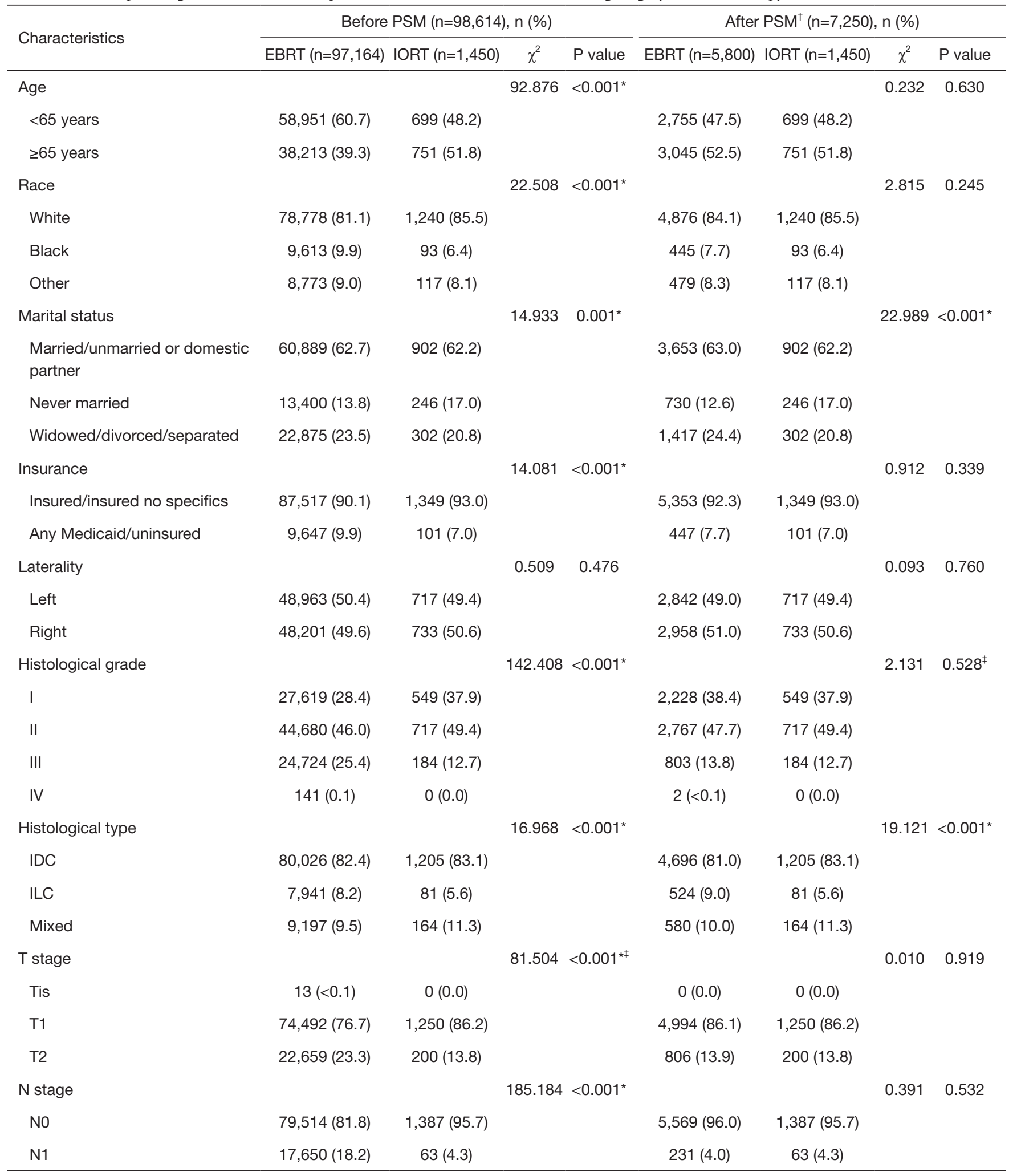

Table 3 (continued) 
Table 3 (continued)

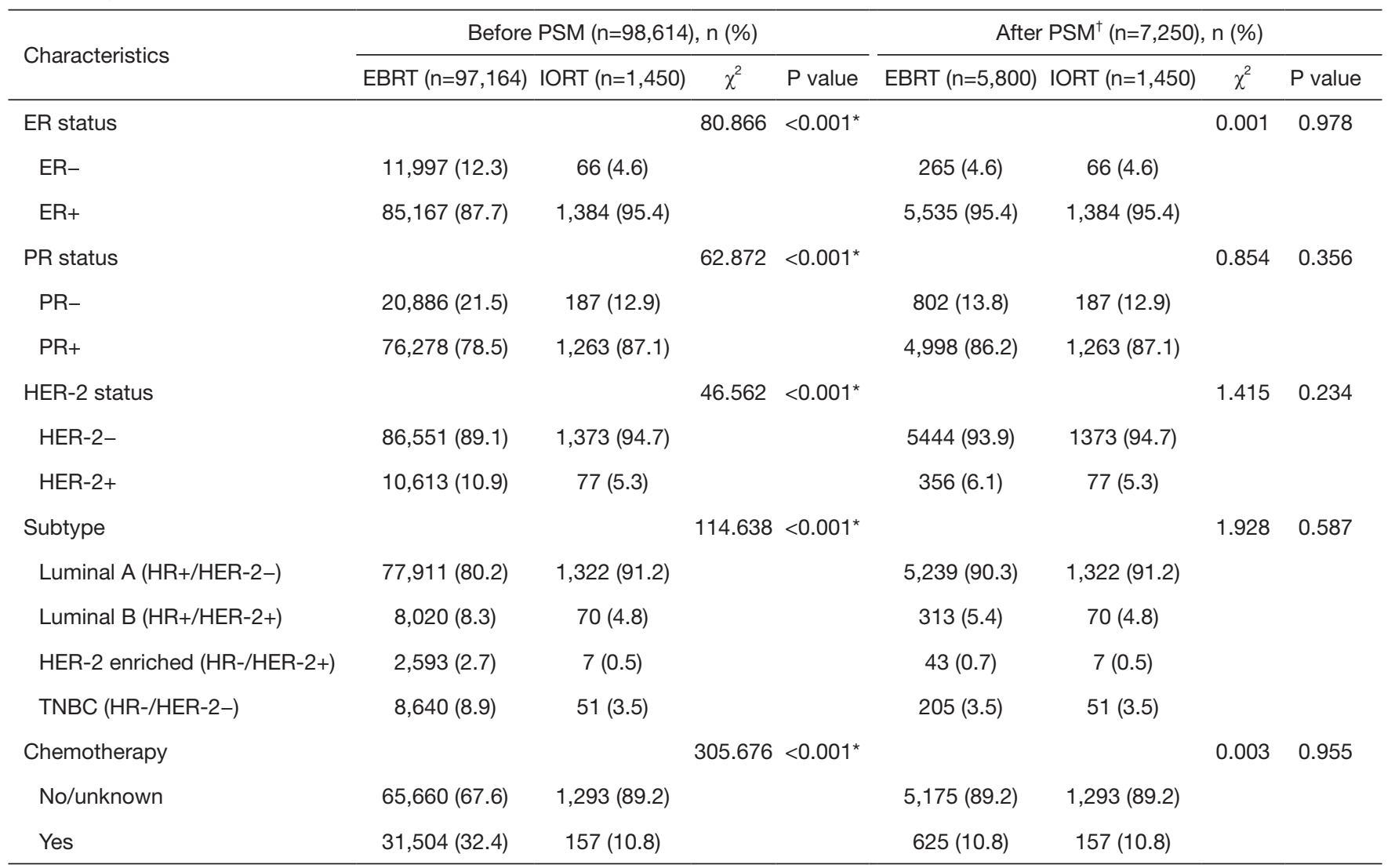

${ }^{*}, \mathrm{P} \leq 0.05$ indicated statistical significance. ${ }^{\dagger}$, matched by age, race, marital status, insurance, laterality, histological grade, histological type, T stage, N stage, ER status, PR status, HER-2 status, subtype, and chemotherapy. ${ }^{\ddagger}$, Monte Carlo method was adopted because the minimum expected count $<1$, or more than $20 \%$ cells have expected count $<5$; Pearson chi-square test was used for all others. PSM, propensity score matching; EBRT, external beam radiotherapy; IORT, intraoperative radiotherapy.

guidelines version 2019 strongly recommended wholebreast irradiation with or without a boost to tumor bed for patients after BCS (3). This recommendation was supported by EBCTCG's meta-analysis, which demonstrated that patients who received whole-breast irradiation had a $16 \%$ reduction in 10-year risk of recurrence and a $4 \%$ reduction in 15-year risk of breast cancer death than those who did not (4). In our study, we found that age $\geq 65$, poor marital status, lack of medical insurance, histological grade III/ $\mathrm{IV}$, high $\mathrm{T}$ stage, high $\mathrm{N}$ stage, and TNBC were associated with a poorer OS/BCSS, whereas ER-positive and PRpositive were associated with an improved OS/BCSS. Several studies in related fields demonstrated similar results. A retrospective study by Kim et al. indicated that high $\mathrm{T}$ stage and positive lymph node metastasis were associated with a worse OS, while positive lymph node metastasis and high histological grade were associated with a poor local recurrence-free survival (LRFS) for breast cancer treated with BCS and radiotherapy (9). Wang et al. reviewed cases receiving postmastectomy radiotherapy (PMRT) and found that TNBC and high N stage were independent predictors of a poor LRFS (8). Another retrospective study by Lei et al. indicated that the high $\mathrm{T}$ stage was associated with a poorer OS, whereas ER-positive tumors were associated with an increased OS for early breast cancer treated with BCS and radiotherapy (22). The results of a recent randomized controlled trial indicated that large tumor size, high tumor grade, more positive nodes, and TNBC were associated with a higher IBTR rate, whereas ER-positive was associated with a lower one (20). The prognostic factors found in our study were consistent with those of previous studies, enhancing the reliability of our results. Besides, the results of our study indicated that marital status and insurance status, two factors that were rarely involved in studies before, were independent 

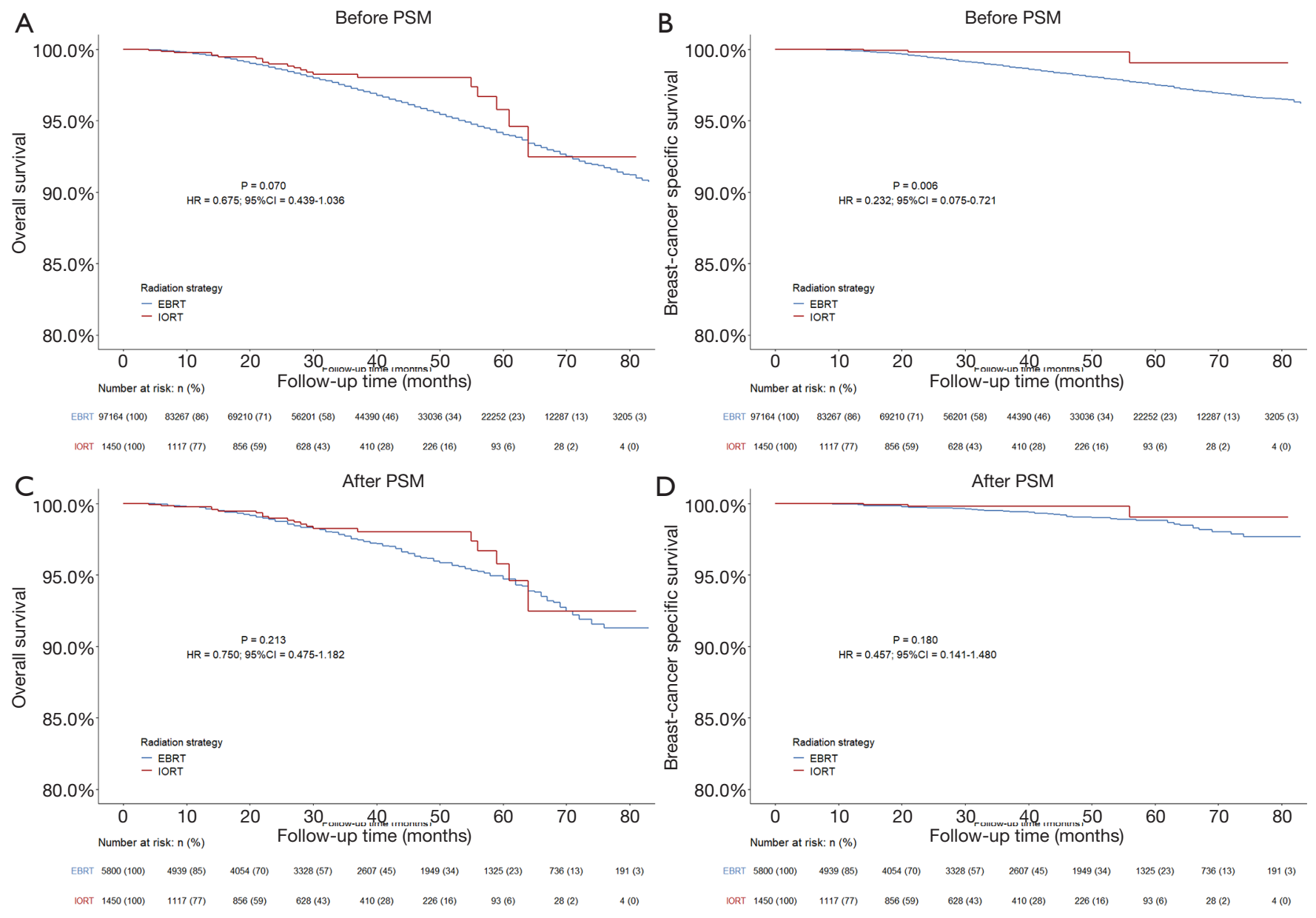

Figure 2 Kaplan-Meier survival curves in early breast cancer patients who underwent EBRT and IORT for overall survival before (A) and after (C) PSM, and breast-cancer specific survival before (B) and after (D) PSM. PSM, propensity score matching; EBRT, external beam radiotherapy; IORT, intraoperative radiotherapy.

prognostic factors for early breast cancer treated with BCS and radiotherapy. We believed that marital status could affect the emotional state of patients, and insurance status was closely related to economic condition, hence these two factors had an impact on the survival of patients. The impact of these sociological factors on the prognosis should be paid more attention in future studies.

Radiotherapy plays an essential part in BCS, however whole-breast EBRT precludes a considerable proportion of patients from receiving the complete course of radiation treatment due to the long course. Combining the advantages of hypofractionated radiation and APBI, the strategy of IORT allows a shorter therapy course and a reduced volume of irradiated breast (16). Furthermore, IORT is a more cost-effective strategy for early breast cancer, providing greater quality-adjusted life years (QALY) at a decreased cost compared with whole-breast EBRT (23-25). Despite all these advantages of IORT, clinicians may still think that IORT is not comparable to EBRT, because of concerns that it may lead to a higher recurrence rate. The emergence of two randomized controlled equivalence trails, the TARGIT-A trial and the ELIOT trial, dispelled their scruples. The TARGIT-A trial went through a median follow-up period of 29 months, demonstrating that the 5-year LRR in single-dose targeted intraoperative radiotherapy (TARGIT) group was within the non-inferiority criteria compared with the EBRT group (19). The ELIOT trial went through a median follow-up of 5.8 years, suggesting that compared with the EBRT group, the 5-year IBTR rate in the intraoperative 


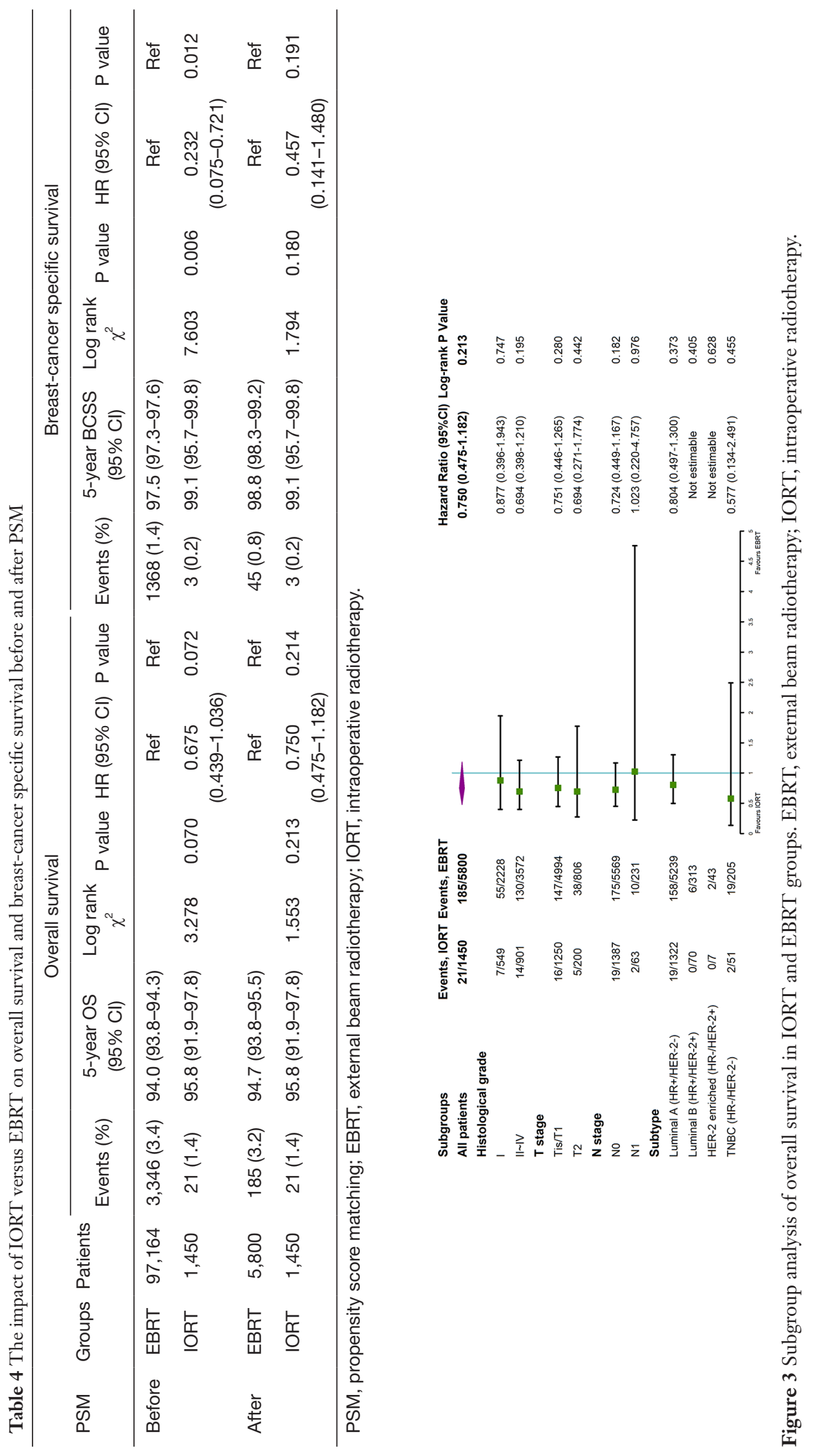




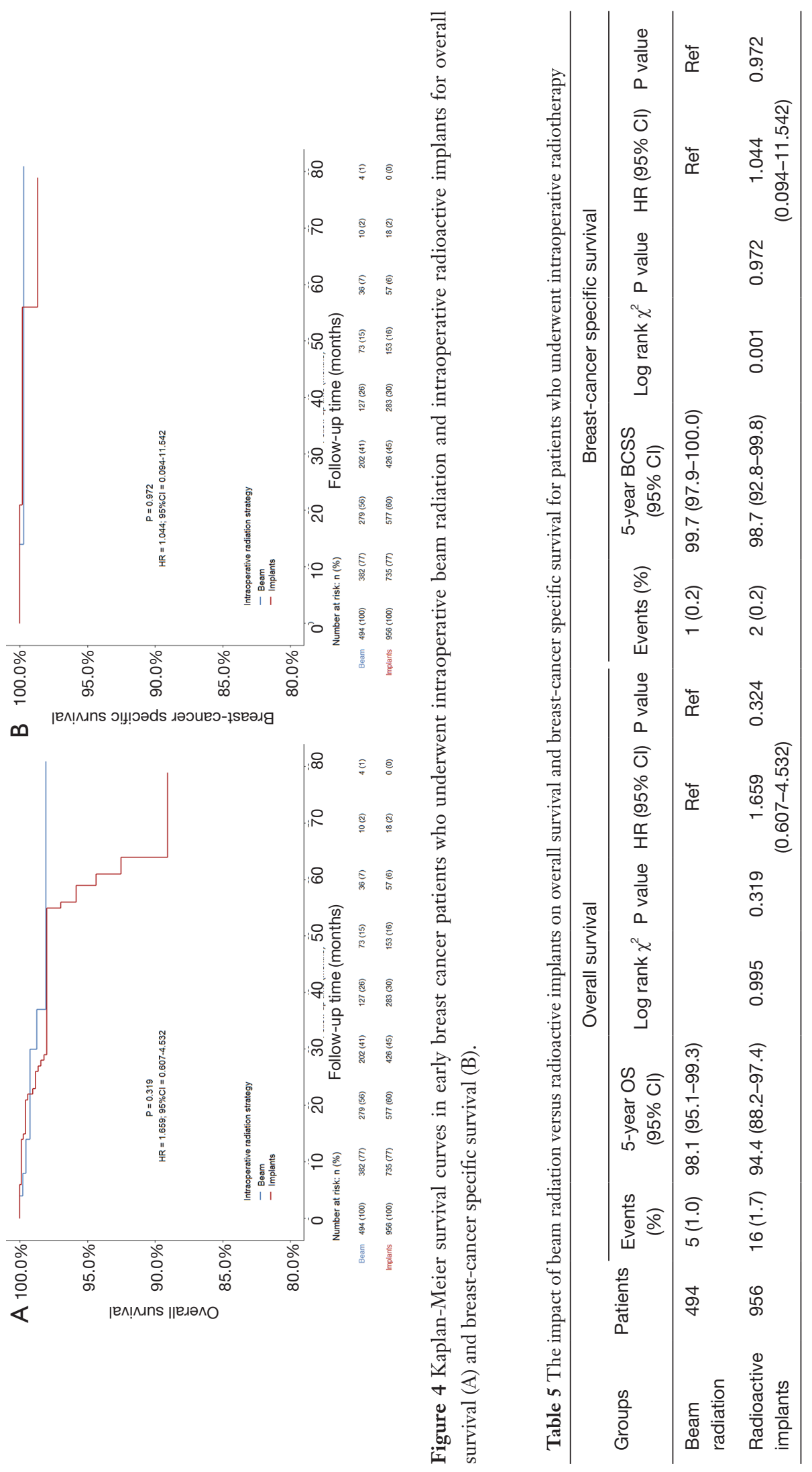


electron radiotherapy (IOERT) group was also within the non-inferiority criteria preset in the trial (20). However, there were some limitations in the two randomized controlled trials above. The TARGIT-A trial only went through a median follow-up period of 29 months (26), and the number of patients in the IORT group of the ELIOT trial was insufficient, which was only 651 (27). Besides, the main outcome indicator of the two studies was the recurrence rate, and there was little discussion on the survival rate. In our study, we focused on the survival rate in the IORT and the EBRT group, with a median follow-up period of 36 months, up to 1,450 patients included in the IORT group, and the baseline clinicopathological factors fully matched by PSM. The results of our study proved that IORT wasn't inferior to EBRT in terms of short-term survival, no matter on OS or BCSS. To summarize, IORT allows a shorter therapy course and a reduced radiation volume, although it leads to an acceptable increase in recurrence rate, there is no difference between IORT and EBRT in terms of short-term survival rate, which fully proves the advantages of IORT and shows that it is a good alternative to EBRT for early breast cancer patients who were unable to complete the long-term postoperative radiation treatment. So how to find the suitable population of patients for IORT? Some researchers believed that patients with high ER/PR, low HER-2, low ki67 index, and low tumor grade were suitable for IORT, but there was still a lack of effective data to prove it $(28,29)$. In our study, no prognostic difference was observed in survival between IORT and EBRT groups in subgroup analysis classified by histological grade, T stage, $\mathrm{N}$ stage, and molecular subtype, indicating that the suitable population for IORT was not clear yet. Furthermore, no significant difference was observed in survival between intraoperative beam radiation and intraoperative radioactive implants, indicating that beam radiation and radioactive implants were both ideal alternatives for patients who chose IORT.

The major strength of this study was that we collected data based on the national population and included many clinicopathological factors to explore the prognostic factors of early breast cancer treated with BCS and radiotherapy. Besides, different from the previous studies on the recurrence rate, we took survival rate as the prognostic indicator, went through a long period of follow-up, with 1,450 patients included in the IORT group and the baseline clinicopathological factors fully matched with the help of PSM owing to the huge sample size obtained from the SEER database. We would like to acknowledge some limitations of our study: (I) the SEER database didn't record information about recurrence and irradiation dose, so the recurrence rate and irradiation dose couldn't be incorporated into analyses. It is important to design rigorous studies with our data and incorporate these factors into analyses in the future. (II) The SEER database didn't have a comprehensive record of radiotherapy, chemotherapy and endocrine therapy, so we were unable to extract much data about the dose and frequency of radiotherapy, the regimen of chemotherapy, and endocrine therapy, and we were unable to distinguish between nonchemotherapy and chemotherapy unknown. These factors that couldn't be included in the study might have an impact on prognosis. (III) Patients with Tis-2 N0-1 M0 breast cancer in this study had a relatively good prognosis, which was beneficial to produce a non-inferior result. Therefore, the current study results should be interpreted with caution. (IV) Although the study indicated that IORT is noninferior in prognosis, there was a lack of data on the side effects of IORT. (V) We excluded samples with incomplete demographic and clinicopathological information, which might contribute to the selection bias. (VI) In our study, a median of 36 months of follow-up was still not long enough compared with the development of the disease. (VII) As we only collected data from the SEER database, further work is required to address the universality of these results.

\section{Conclusions}

Our study demonstrated that age $\geq 65$, poor marital status, lack of medical insurance, histological grade III/IV, high $\mathrm{T}$ stage, high $\mathrm{N}$ stage, and TNBC were associated with a decreased OS/BCSS, whereas ER-positive and PR-positive were associated with an improved OS/BCSS for early breast cancer treated with BCS and radiotherapy. IORT allows a shorter therapy course and a reduced radiation volume, although it leads to an acceptable increase in recurrence rate, there is no difference between IORT and EBRT in terms of short-term survival rate, which fully proves the advantages of IORT and shows that it is a good alternative to EBRT for early breast cancer patients who were unable to complete the long-term postoperative radiation treatment. Beam radiation and radioactive implants are both ideal alternatives for patients who choose IORT.

\section{Acknowledgments}

We thank the staff at the SEER database. 
Funding: This work was supported by the Beijing Medical Award Foundation (No. YXJL-2016-0040-0065).

\section{Footnote}

Reporting Checklist: The authors have completed the STROBE reporting checklist. Available at http://dx.doi. org/10.21037/tcr-20-2441

Peer Review File: Available at http://dx.doi.org/10.21037/tcr20-2441

Conflicts of Interest: All authors have completed the ICMJE uniform disclosure form (available at http://dx.doi. org/10.21037/tcr-20-2441). The authors have no other conflicts of interest to declare.

Ethical Statement: The authors are accountable for all aspects of the work in ensuring that questions related to the accuracy or integrity of any part of the work are appropriately investigated and resolved. The study was conducted in accordance with the Declaration of Helsinki (as revised in 2013). Informed consent is unnecessary because SEER data are retrospective and unidentified.

Open Access Statement: This is an Open Access article distributed in accordance with the Creative Commons Attribution-NonCommercial-NoDerivs 4.0 International License (CC BY-NC-ND 4.0), which permits the noncommercial replication and distribution of the article with the strict proviso that no changes or edits are made and the original work is properly cited (including links to both the formal publication through the relevant DOI and the license). See: https://creativecommons.org/licenses/by-nc-nd/4.0/.

\section{References}

1. Harbeck N, Gnant M. Breast cancer. Lancet 2017;389:1134-50.

2. Laronga C, Lewis JD, Smith PD. The changing face of mastectomy: an oncologic and cosmetic perspective. Cancer Control 2012;19:286-94.

3. Telli ML, Gradishar WJ, Ward JH. NCCN Guidelines Updates: Breast Cancer. J Natl Compr Canc Netw 2019;17:552-5.

4. Darby S, McGale P, Correa C, et al. Effect of radiotherapy after breast-conserving surgery on 10-year recurrence and 15-year breast cancer death: meta-analysis of individual patient data for 10,801 women in 17 randomised trials. Lancet 2011;378:1707-16.

5. Clarke M, Collins R, Darby S, et al. Effects of radiotherapy and of differences in the extent of surgery for early breast cancer on local recurrence and 15 -year survival: an overview of the randomised trials. Lancet 2005;366:2087-106.

6. Veronesi U, Cascinelli N, Mariani L, et al. Twentyyear follow-up of a randomized study comparing breastconserving surgery with radical mastectomy for early breast cancer. N Engl J Med 2002;347:1227-32.

7. Fisher B, Anderson S, Bryant J, et al. Twenty-year followup of a randomized trial comparing total mastectomy, lumpectomy, and lumpectomy plus irradiation for the treatment of invasive breast cancer. $\mathrm{N}$ Engl J Med 2002;347:1233-41.

8. Wang J, Luo J, Jin K, et al. Biological subtype predicts locoregional recurrence after postmastectomy radiotherapy in Chinese breast cancer patients. Cancer Med 2020;9:2427-34.

9. Kim KJ, Huh SJ, Yang JH, et al. Treatment results and prognostic factors of early breast cancer treated with a breast conserving operation and radiotherapy. Jpn J Clin Oncol 2005;35:126-33.

10. Nair VJ, Caudrelier JM. Hypofractionated radiotherapy for elderly breast cancer patients: from early stages disease to local palliation for unresectable disease. Transl Cancer Res 2020;9:S189-S196.

11. Whelan TJ, Pignol JP, Levine MN, et al. Long-term results of hypofractionated radiation therapy for breast cancer. N Engl J Med 2010;362:513-20.

12. Owen JR, Ashton A, Bliss JM, et al. Effect of radiotherapy fraction size on tumour control in patients with early-stage breast cancer after local tumour excision: long-term results of a randomised trial. Lancet Oncol 2006;7:467-71.

13. Murray Brunt A, Haviland JS, Wheatley DA, et al. Hypofractionated breast radiotherapy for 1 week versus 3 weeks (FAST-Forward): 5 -year efficacy and late normal tissue effects results from a multicentre, non-inferiority, randomised, phase 3 trial. Lancet 2020;395:1613-26.

14. Tann AW, Hatch SS, Joyner MM, et al. Accelerated partial breast irradiation: Past, present, and future. World J Clin Oncol 2016;7:370-9.

15. Chen KW, Hsu HT, Lin JF, et al. Adjuvant whole breast radiotherapy with simultaneous integrated boost to tumor bed with intensity modulated radiotherapy technique in elderly breast cancer patients. Translational Cancer Research 2020;9:S12-S22. 
16. Kalakota K, Small W Jr. Intraoperative radiation therapy techniques and options for breast cancer. Expert Rev Med Devices 2014;11:265-73.

17. Kahan Z, Csenki M, Varga Z, et al. The risk of early and late lung sequelae after conformal radiotherapy in breast cancer patients. Int J Radiat Oncol Biol Phys 2007;68:673-81.

18. Darby SC, McGale P, Taylor CW, et al. Long-term mortality from heart disease and lung cancer after radiotherapy for early breast cancer: prospective cohort study of about 300,000 women in US SEER cancer registries. Lancet Oncol 2005;6:557-65.

19. Vaidya JS, Wenz F, Bulsara M, et al. Risk-adapted targeted intraoperative radiotherapy versus wholebreast radiotherapy for breast cancer: 5-year results for local control and overall survival from the TARGIT-A randomised trial. Lancet 2014;383:603-13.

20. Veronesi U, Orecchia R, Maisonneuve P, et al. Intraoperative radiotherapy versus external radiotherapy for early breast cancer (ELIOT): a randomised controlled equivalence trial. Lancet Oncol 2013;14:1269-77.

21. Vanoni V, Bou Selman S, Mussari S, et al. External Beam Radiation Therapy Versus Intraoperative Radiation Therapy for Breast-Conserving Therapy: A Large SingleInstitution Matched-Pair Evaluation. Int J Radiat Oncol Biol Phys 2014;90:S261.

22. Lei J, Wang Y, Bi Z, et al. Intraoperative radiotherapy (IORT) versus whole-breast external beam radiotherapy (EBRT) in early stage breast cancer: results from SEER database. Jpn J Radiol 2020;38:85-92.

Cite this article as: Sun MS, Liu HJ, Liu YH, Xu L, Ye JM. Intraoperative radiotherapy versus whole-breast external beam radiotherapy, and other factors associated with the prognosis of early breast cancer treated with breast-conserving surgery and radiotherapy: a retrospective study from SEER database. Transl Cancer Res 2020;9(11):7125-7139. doi: 10.21037/tcr-20-2441
23. Vaidya A, Vaidya P, Both B, et al. Health economics of targeted intraoperative radiotherapy (TARGIT-IORT) for early breast cancer: a cost-effectiveness analysis in the United Kingdom. BMJ Open 2017;7:e014944.

24. Patel R, Ivanov O, Voigt J. Lifetime cost-effectiveness analysis of intraoperative radiation therapy versus external beam radiation therapy for early stage breast cancer. Cost Eff Resour Alloc 2017;15:22.

25. Alvarado MD, Mohan AJ, Esserman LJ, et al. Costeffectiveness analysis of intraoperative radiation therapy for early-stage breast cancer. Ann Surg Oncol 2013;20:2873-80.

26. Silverstein MJ, Fastner G, Maluta $S$, et al. Intraoperative radiation therapy: a critical analysis of the ELIOT and TARGIT trials. Part 2--TARGIT. Ann Surg Oncol 2014;21:3793-9.

27. Silverstein MJ, Fastner G, Maluta S, et al. Intraoperative radiation therapy: a critical analysis of the ELIOT and TARGIT trials. Part 1--ELIOT. Ann Surg Oncol 2014;21:3787-92.

28. Guenzi M, Bonzano E, Corvo R, et al. Comparison of Local Recurrence Among Early Breast Cancer Patients Treated With Electron Intraoperative Radiotherapy vs Hypofractionated Photon Radiotherapy an Observational Study. Front Oncol 2018;8:207.

29. Kolberg HC, Loevey G, Akpolat-Basci L, et al. Targeted Intraoperative Radiotherapy Tumour Bed Boost During Breast Conserving Surgery after Neoadjuvant Chemotherapy in HER2 Positive and Triple Negative Breast Cancer. Rev Recent Clin Trials 2017;12:93-100. 